\title{
Modelos de sistemas integrados de gestión para pequeñas, medianas y grandes empresas*
}

[Artículos de revisión]

\author{
Luz Evely Quintero Becerra** \\ Camila Andrea Rodríguez Díaz ${ }^{* * *}$ \\ Magda Viviana Monroy Silva ****
}

Recibido: 11 de noviembre de 2020

Revisado: 23 de enero de 2021

Aceptado: 13 de marzo de 2021

Citar como:

Quintero Becerra, L. E., Rodríguez Díaz, C. A. y Monroy Silva, M. V. (2021). Modelos

de sistemas integrados de gestión para pequeñas, medianas y grandes

empresas. Signos, Investigación en Sistemas de Gestión, 13(2).

https://doi.org/10.15332/24631140.6673

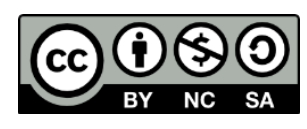

\section{Resumen}

El presente artículo de revisión sistemática de literatura se desarrolló con

el objetivo de identificar los principales modelos de sistemas integrados

\footnotetext{
* Artículo de revisión.

** Químico industrial del grupo Control de Calidad Dirección Administrativa y Financiera de la Policía. Correo electrónico: luzquinterob@usantotomas.edu.co; ORCID: https://orcid.org/00000001-7594-9710; CVLAC:

https://scienti.minciencias.gov.co/cvlac/visualizador/generarCurriculoCv.do?cod rh=0001776058

${ }^{* * *}$ Ingeniera industrial. Instituto Distrital de Turismo. Correo electrónico: camilarodriguezd@usantotomas.edu.co; ORCID: https://orcid.org/0000-0002-8227-9740;

CVLAC: https://scienti.minciencias.gov.co/cvlac/visualizador/generarCurriculoCv.do?cod rh $=000$ 1776050

**** Magíster en Ingeniería Industrial por la Universidad Santo Tomás. Correo: magdamonroy@usantotomas.edu.co; ORCID: https://orcid.org/0000-0002-6185-5999; CVLAC: https://scienti.minciencias.gov.co/cvlac/visualizador/generarCurriculoCv.do?cod rh $=0000003418$
}

Signos, Investigación en Sistemas de Gestión

ISSN: 2145-1389 | e-ISSN: 2463-1140 | DOI: https://doi.org/10.15332/24631140

Vol. 13 N.० 2 | julio-diciembre de 2021 
de gestión (SIG) para pequeñas, medianas y grandes empresas; esto por medio de un análisis bibliométrico que permitió determinar cuáles son los modelos utilizados para la integración de sistemas en cada uno de los tipos de empresas analizados. Se entienden los SIG como un conjunto único de procesos interconectados que incluyen dos o más sistemas de gestión; estos fueron explorados a través de investigación de tipo cualitativo y descriptivo, en la búsqueda, recolección, depuración y análisis de información de las bases de datos Science Direct, Scopus, Redalyc y Google Scholar. Con base en los resultados, se encontró que las estrategias de integración se diseñan de forma general y no enfocadas en tamaños de organizaciones, es decir, que estas son implementadas para cualquier tamaño de empresa. Como conclusiones se evidenció que se enfocan en sectores como energía, combustibles, cerámica, alimentos, medicamentos, metalmecánico, entre otros.

Palabras clave: sistemas integrados de gestión, estrategias de integración, pequeñas empresas, medianas empresas, grandes empresas.

\section{Models of integrated management systems for small, medium and large companies}

\section{Abstract}

This systematic literature review article was developed aiming at identifying the main models of integrated management systems (IMS) for small, medium and large companies; this by means of a bibliometric analysis that made it possible to determine which are the models used for the integration of systems in each of the types of companies analyzed. IMSs are understood as a single set of interconnected processes wherein two or more management systems are included; These were explored through qualitative and descriptive research, in the search, collection, debugging and analysis of information from the Science Direct, Scopus, Redalyc and Google Scholar databases. Based on the results, it was found that integration strategies are generaly designed instead of being focused on organization sizes, that is, they are implemented for any size of 
company. As conclusions, it was evidenced that they focus on sectors such as energy, fuels, ceramics, food, medicine, metalworking, among others.

Keywords: integrated management systems, integration strategies, small companies, medium-sized companies, large companies.

\section{Modelos de sistemas integrados de gestão para pequenas, médias e grandes empresas}

\section{Resumo}

Este artigo de revisão bibliogáfica sistemática foi desenvolvido visando identificar os principais modelos de sistemas integrados de gestão (SIG) para pequenas, médias e grandes empresas; isto através de uma análise bibliométrica que permitiu determinar quais os modelos utilizados para a integração de sistemas em cada um dos tipos de empresas analisados. Os SIG são entendidos como um conjunto único de processos interligados que incluem dois ou mais sistemas de gestão; estes foram explorados através de pesquisa qualitativa e descritiva, pesquisa, coleta, depuração e análise de informações das bases de dados Science Direct, Scopus, Redalyc e Google Scholar. Com base nos resultados, verificou-se que as estratégias de integração são projetadas de forma geral e não focalizadas no tamanho das organizações, isto é, são implementadas para qualquer tamanho de empresa. Como conclusões, foi evidenciado que elas estão focadas em setores como energia, combustíveis, cerâmica, alimentos, medicamentos, metalurgia, entre outros.

Palavras-chave: sistemas integrados de gestão, estratégias de integração, pequenas empresas, médias empresas, grandes empresas.

\section{Introducción}

Los sistemas de gestión se entienden como aquellos que permiten obtener una mejora continua de sus procesos al hacer uso de técnicas, herramientas, programas y tecnologías enfocados en lograr la satisfacción 
del cliente (Oliveros y Soto, 2016). También son concebidos como un conjunto único de procesos interconectados que comparten una reserva determinada de recursos humanos, de información, infraestructura y financieros, para lograr los objetivos relacionados con la satisfacción de las partes interesadas (Stamou, 2003).

En los últimos años muchas empresas han adoptado sistemas de gestión de calidad (SIG), dado que la calidad parece ser un requisito fundamental para la competitividad (Stamou, 2003). Además, recientemente las cuestiones ambientales y la legislación sobre seguridad y salud en el trabajo han introducido procedimientos de control específicos adicionales (sistemas de gestión ambiental y la seguridad y salud en el trabajo SGSST-) (Mosquera y Vásquez, 2015; Lleras, 2017; Pohludka y Štverková, 2019; Díaz y Rodríguez-Rojas, 2016, Stamou, 2003). Estos sistemas de gestión de calidad, ambiental, seguridad y salud en el trabajo son gestionados por separado por diferentes áreas funcionales de las organizaciones (Mosquera y Vásquez, 2015; Lleras, 2017; Stamou, 2003); lo cual genera un exceso de tramites, alto costo para las organizaciones, y se duplican los esfuerzos en la elaboración y aplicación de estas normas.

Si la organización adopta una norma de un sistema de gestión, los documentos dispuestos para esa admisión pueden utilizarse para otra norma de sistema de gestión con modificaciones específicas (Khanna et ál., 2009). Las sinergias y los numerosos puntos de contacto entre los sistemas mencionados han dado lugar a los sistemas integrados de gestión (SIG) como una forma de cumplir los requisitos de gestión de la calidad, gestión ambiental y gestión de la seguridad y salud en el trabajo (Stamou, 2003; Nunhes y Oliveira, 2020; Khanna et ál., 2009). Particularmente se evidencian beneficios como la disminución de costos, mejora de la imagen organizacional, aumento del grado de satisfacción del cliente y una mayor motivación de los colaboradores (Khanna et ál., 2009; Mosquera y 
Vásquez, 2015; Lleras, 2017; Pohludka y Štverková, 2019; Díaz y Rodríguez-Rojas, 2016; Stamou, 2003).

Los beneficios asociados con el establecimiento del SIG pueden ser: a) económicos, como la reducción del riesgo de sanciones ambientales, los costos de actividades de rehabilitación, de recursos y materiales de desecho, disminución de documentación, capacitación; b) organizacionales, como mejores oportunidades de trabajo, incremento del mercado, ambiente laboral positivo y operaciones internas bien definidas; y c) ambientales, como la reducción del impacto sobre el medio ambiente, los recursos naturales y la comunidad en general (Khanna et ál., 2009; Díaz y Rodríguez-Rojas, 2016). En este sentido, diversos autores se han enfocado en el estudio y diseño de SIG para pequeñas y medianas empresas (pymes) (Parada y Paredes, 2014; Gómez, 2009; López, 2009; Brenes et ál., 2017; Stamou, 2003) con resultados positivos, no solo asociados a las organizaciones, sino al cumplimiento de objetivos macroeconómicos y al desempeño financiero que fomentan el bienestar de la sociedad.

La aplicación de sistemas de gestión, y por lo tanto del SIG, no solo es técnicamente apropiada para las pymes, sino que puede ser sustancialmente más fácil para ellas que para las grandes empresas, en razón de que cuentan con un menor número de procesos y de áreas que se deban documentar (Stamou, 2003). Además, las estrategias de integración como estructura de alto nivel, por procesos, ciclo PHVA, se han establecido de manera general, es decir, no han sido estandarizadas por tipo de empresa.

Por otro lado, aunque la literatura evidencia ventajas al integrar los SIG, se sostiene que las pymes pueden encontrar muchas fuentes de limitación, como los costos imprevistos necesarios y las aptitudes del personal, la 
ausencia de integración de los sistemas sin problemas o la falta de recompensas del mercado (Stamou, 2003) en el proceso de implementación de los SIG.

En cuanto a las pymes colombianas, la decisión de incluir la certificación en las normas ISO tiene una causa asociada a las exigencias del mercado, dado que algunas medianas y grandes empresas exigen a sus proveedores contar con estas certificaciones (Ospina y Rivera, 2013), mientras que en las grandes empresas tiene como fin mejorar la integración de sus áreas y la disminución de los costos de sus sistemas de gestión (Díaz y RodríguezRojas, 2016).

En relación con todo lo anterior, este artículo tiene como propósito realizar un análisis bibliométrico para identificar y describir los modelos existentes de SIG para pequeñas, medianas y grandes empresas, lo cual es importante en el posicionamiento y reconocimiento dentro de los mercados nacionales e internacionales. Dado que, como se mencionó anteriormente, la implementación correcta de los sistemas integrados de gestión permite aumentar la productividad, mejorar la competitividad empresarial, armonizar y documentar mejor cada actividad, proceso o procedimiento, desarrollado al interior de las organizaciones, conocer las necesidades y requisitos de los productos o servicios que se entregan a los clientes externos, logrando una percepción efectiva tanto de los servicios prestados como de la imagen de la empresa (Mosquera y Vásquez, 2015). El presente artículo cuenta con cuatro secciones en su desarrollo. La primera es la introducción. Seguida de la metodología, en la cual se describe el proceso de búsqueda y depuración en la fase de revisión de literatura, así como el análisis, con base en variables bibliométricas y técnicas. En la tercera, se presentan los resultados y la discusión, de acuerdo con las variables relacionadas con modelos de SIG, en pequeñas, 
medianas y grandes empresas y las estrategias de integración. Finalmente, se presentan las conclusiones pertinentes que servirán de insumo para estudios posteriores.

\section{Metodología}

\section{Tipo de estudio}

La presente investigación se enmarca en un estudio de carácter cualitativo

y descriptivo, dado que se guía su desarrollo en la descripción de variables definidas para la investigación (Hernández et ál., 2010). También está enmarcada en un estudio de tipo bibliométrico, por cuanto se investigan y analizan artículos relacionados con modelos de SIG en pequeñas, medianas y grandes empresas, por medio de la identificación y cuantificación de diferentes variables. Para tal efecto, se emplearon herramientas bibliométricas que son fundamentales para la captación de información, análisis y toma de decisiones (Hernández et ál., 2010).

La pregunta de investigación planteada para desarrollar el objetivo del presente artículo fue: ¿cuáles son los modelos de sistemas integrados de gestión más usados en pequeñas, medianas y grandes empresas?

\section{Fundamentación de la metodología}

El desarrollo de la bibliometría se inició en 1923, por Hulme con términos como "statistical bibliography" cuando se hizo referencia al proceso de reunión e interpretación de estadísticas relativas a libros y periódicos; sin embargo, quien acuñó el término fue Paul Otlet en 1934, al usar el término francés bibliometrie, quien la definió como una parte de la bibliología que se ocupa de la medida o cantidad aplicada a los libros (Michán y MuñozVelasco, 2013). Sin embargo, a la ciencia actual se le reconoce gracias a Alain Pitchard, quien acuñó el término en 1969 e hizo evidente este 
término al definirlo como el proceso de depuración, recopilación y análisis de grandes cantidades de bibliografía con el objetivo de identificar patrones o tendencias (Michán y Muñoz, 2013).

\section{Proceso de recolección de información}

A partir de la pregunta de investigación se definieron los siguientes términos de búsqueda: "models", "integrated management systems", "small companies", "medium companies" y "large companies". A partir de estos términos de búsqueda se diseñaron las ecuaciones de búsqueda:

- " "models" and "integrated management systems".

- " integrated management systems" and "small companies".

- "integrated management systems" and "medium companies".

- " integrated management systems" and "large companies".

- "models" and "integrated management systems" and "companies".

Se seleccionaron 4 bases de datos para la realización de la búsqueda: Science Direct, Scopus, Redalyc y Google Scholar. De acuerdo con lo anterior, se presentan los resultados obtenidos (ver tabla 1):

Tabla 1. Resultados de las búsquedas realizadas Science Direct

\begin{tabular}{|c|c|c|c|c|}
\hline & $\begin{array}{l}\text { Science } \\
\text { Direct }\end{array}$ & $\begin{array}{c}\text { Filtro } 1 \\
\text { (artícul } \\
\text { os) }\end{array}$ & $\begin{array}{c}\text { Filtro } 2 \\
\text { (open } \\
\text { access) }\end{array}$ & $\begin{array}{l}\text { Filtro } 3 \\
\text { (título) }\end{array}$ \\
\hline "models" and "integrated management systems". & 964 & 52 & 3 & 0 \\
\hline $\begin{array}{l}\text { "integrated management systems" and "small } \\
\text { companies". }\end{array}$ & 41 & 25 & 3 & 2 \\
\hline $\begin{array}{l}\text { "integrated management systems" and "medium } \\
\text { companies". }\end{array}$ & 11 & 11 & 10 & 2 \\
\hline $\begin{array}{c}\text { "integrated management systems" and "large } \\
\text { companies". }\end{array}$ & 83 & 66 & 11 & 1 \\
\hline $\begin{array}{l}\text { "models" and "integrated management systems" } \\
\text { and "companies". }\end{array}$ & 596 & 460 & 95 & 6 \\
\hline
\end{tabular}

Fuente: elaboración propia (2021), fundamentada en información de bases de datos. 
Tabla 2. Resultado de las búsquedas realizadas Scopus

\begin{tabular}{|c|c|c|c|c|}
\hline & $\begin{array}{l}\text { Scop } \\
\text { us }\end{array}$ & $\begin{array}{l}\text { Filtro } 1 \\
\text { (artículos) }\end{array}$ & $\begin{array}{l}\text { Filtro } 2 \\
\text { (open } \\
\text { access) }\end{array}$ & $\begin{array}{l}\text { Filtro } 3 \\
\text { (titulo) }\end{array}$ \\
\hline "models" and "integrated management systems". & 333 & 182 & 40 & 16 \\
\hline $\begin{array}{c}\text { “integrated management systems" and "small } \\
\text { companies". }\end{array}$ & 2 & 1 & 0 & 0 \\
\hline $\begin{array}{l}\text { "integrated management systems" and "medium } \\
\text { companies". }\end{array}$ & 4 & 4 & 3 & 2 \\
\hline $\begin{array}{c}\text { “integrated management systems" and “large } \\
\text { companies”. }\end{array}$ & 8 & 4 & 4 & 3 \\
\hline $\begin{array}{c}\text { "models" and "integrated management systems" } \\
\text { and "companies". }\end{array}$ & 87 & 53 & 14 & 1 \\
\hline
\end{tabular}

Fuente: elaboración propia (2021), fundamentada en información de bases de datos.

Tabla 3. Resultados de las búsquedas realizadas Redalyc

\begin{tabular}{|c|c|c|c|c|}
\hline & $\begin{array}{l}\text { Reda } \\
\text { lyc }\end{array}$ & $\begin{array}{l}\text { Filtro } 1 \\
\text { (artículos) }\end{array}$ & $\begin{array}{l}\text { Filtro } 2 \text { (open } \\
\text { access) }\end{array}$ & $\begin{array}{l}\text { Filtro } 3 \\
\text { (titulo) }\end{array}$ \\
\hline $\begin{array}{c}\text { "models" and "integrated management } \\
\text { systems". }\end{array}$ & $\begin{array}{c}875 \\
9\end{array}$ & 5130 & 5130 & 1 \\
\hline $\begin{array}{c}\text { "integrated management systems" and "small } \\
\text { companies". }\end{array}$ & 2 & 2 & 1 & 0 \\
\hline $\begin{array}{l}\text { "integrated management systems" and } \\
\text { "medium companies". }\end{array}$ & 75 & 75 & 75 & 15 \\
\hline $\begin{array}{l}\text { “integrated management systems" and "large } \\
\text { companies". }\end{array}$ & 304 & 295 & 295 & 0 \\
\hline $\begin{array}{l}\text { "models" and "integrated management } \\
\text { systems" and "companies". }\end{array}$ & $\begin{array}{c}139 \\
3\end{array}$ & 1200 & 1200 & 0 \\
\hline $\begin{array}{l}\text { "models" and "integrated management } \\
\text { systems" and "companies". }\end{array}$ & $\begin{array}{c}347 \\
0\end{array}$ & 1110 & 2 & 0 \\
\hline
\end{tabular}

Fuente: elaboración propia (2021), fundamentada en información de bases de datos.

Tabla 4. Resultados de las búsquedas en Google Académico

\begin{tabular}{|c|c|c|c|c|}
\hline & $\begin{array}{c}\text { Google } \\
\text { Académico }\end{array}$ & $\begin{array}{c}\text { Filtro 1 } \\
\text { (artículos) }\end{array}$ & $\begin{array}{c}\text { Filtro 2 (open } \\
\text { access) }\end{array}$ & $\begin{array}{c}\text { Filtro 3 } \\
\text { (titulo) }\end{array}$ \\
\hline “models" and "integrated management \\
\begin{tabular}{c} 
systems". \\
\hline \begin{tabular}{c} 
systom \\
\hline
\end{tabular}
\end{tabular} & 5170 & 4680 & 1404 & 3 \\
\hline
\end{tabular}

Signos, Investigación en Sistemas de Gestión

ISSN: 2145-1389 | e-ISSN: 2463-1140 | DOI: https://doi.org/10.15332/24631140

Vol. 13 N.0 2 | julio-diciembre de 2021 


\begin{tabular}{|c|c|c|c|c|}
\hline & $\begin{array}{c}\text { Google } \\
\text { Académico }\end{array}$ & $\begin{array}{c}\text { Filtro 1 } \\
\text { (artículos) }\end{array}$ & $\begin{array}{c}\text { Filtro 2 (open } \\
\text { access) }\end{array}$ & $\begin{array}{c}\text { Filtro 3 } \\
\text { (titulo) }\end{array}$ \\
\hline $\begin{array}{c}\text { "integrated management systems" and } \\
\text { "small companies". }\end{array}$ & 576 & 254 & 5 & 1 \\
\hline $\begin{array}{c}\text { "integrated management systems" and } \\
\text { "medium companies". }\end{array}$ & 109 & 109 & 44 & 2 \\
\hline $\begin{array}{c}\text { "integrated management systems" and } \\
\text { "large companies". }\end{array}$ & 649 & 579 & 14 & 0 \\
\hline $\begin{array}{c}\text { "models" and "integrated management } \\
\text { systems" and "companies". }\end{array}$ & 3470 & 1110 & 2 & 0 \\
\hline
\end{tabular}

Fuente: elaboración propia (2021), fundamentada en información de bases de datos.

De acuerdo con los filtros realizados, se obtuvo un total de 55 artículos relacionados con el tema de estudio, la depuración de los éstos se muestra en la siguiente figura.

Figura 1. Esquema de depuración de artículos
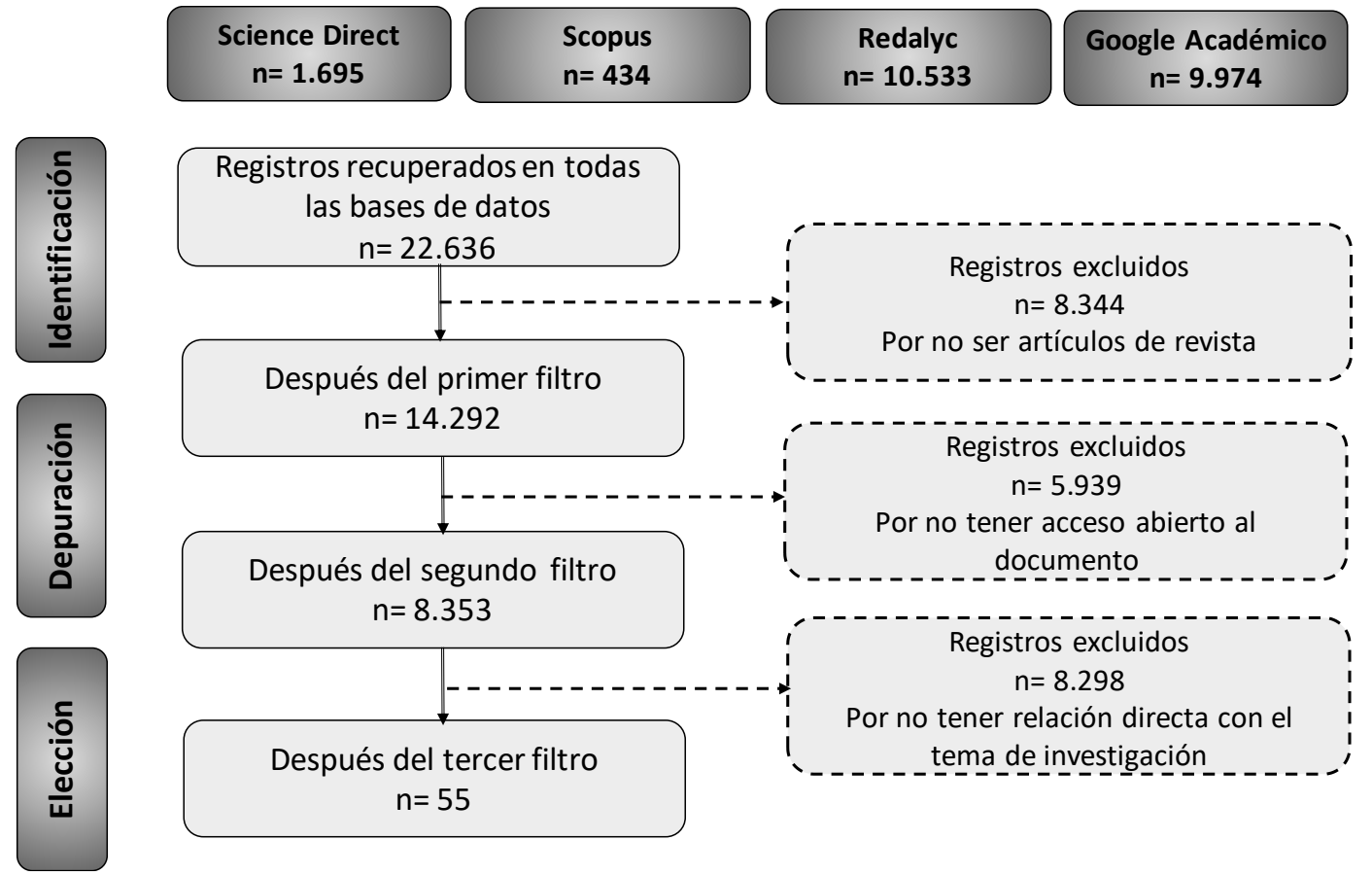

Fuente: elaboración propia (2021).

Los 55 artículos se analizaron según variables bibliométricas como el año de publicación, el tamaño de la empresa y el sector en el que se desarrolla

Signos, Investigación en Sistemas de Gestión

ISSN: 2145-1389 | e-ISSN: 2463-1140 | DOI: https://doi.org/10.15332/24631140

Vol. 13 N.o 2 | julio-diciembre de 2021 
el modelo; además, se describen cualitativamente las siguientes variables técnicas: a) SIG que hace referencia a las diferentes definiciones y conceptualizaciones acerca de los sistemas integrados de gestión de manera general; b) aquellos modelos que se refieren a los artículos que, además, describen modelos particulares para la integración de los sistemas de gestión en cualquier tipo de empresas; y en cuanto a c) los SIG en pequeñas empresas: se refieren a los modelos de SIG enfocados a pequeñas empresas, así como SIG medianas y grandes empresas.

\section{Resultados y discusión}

Dentro de los sistemas que han sido sujetos de integración, se encuentran el sistema de gestión de calidad bajo la norma ISO 9001 y el sistema de gestión ambiental bajo la norma ISO 14001, esta integración puede ayudar a las empresas a mantener la ventaja competitiva y a superar los problemas con las normas de calidad, y a su vez, inducir a las empresas a adoptar buenas prácticas ambientales. De manera general, los modelos y estrategias de implementación de los SIG han sido estudiados por diversos autores en donde se han usado diferentes estrategias de integración como el ciclo PHVA, estructura de alto nivel por procesos, entre otras (Rodríguez y Montenegro, 2019; Khanna et ál., 2009; Alzate-Ibañez et ál., 2018; Mora-Contreras 2019), en razón de que cuentan con un menor número de procesos y de áreas que se deban documentar.

Algunos estudios se han enfocado en discutir las ventajas relacionadas con el aumento de las ventas y de la productividad de las organizaciones, que se derivan del impacto que pueden tener cuando se logra la implementación de las normas ISO en sistemas de gestión (Díaz y Rodríguez-Rojas, 2016). De igual manera, otros estudios han intentado determinar la relación existente entre la implementación de SIG en pymes y la mejora de la productividad, y han encontrado que dentro de los SIG 
la certificación en la norma ISO 14001 Sistema de Gestión Ambiental es la menos utilizada dentro de estas organizaciones (Mosquera y Vásquez, 2015).

Según Oliveros y Soto (2016), los beneficios de la implementación de los SIG en pymes son, por un lado, el recurso humano, en cuanto al perfeccionamiento de sus competencias, la comprensión de sus responsabilidades y la mejora del desempeño laboral. Por el otro, con los procesos y procedimientos, en aspectos como la articulación de auditorías, la estandarización de procesos, planes y programas. Esto debido a que corresponden a la integración de requisitos comunes y aplicables a los sistemas de gestión, bajo las normas de interés.

Otros autores sostienen que la efectividad de los modelos de implementación varía dependiendo del número de sistemas de gestión implementados; es decir, las organizaciones con tres sistemas implementados pueden enfrentar mejor las dificultades en el proceso de integración que afectan al nivel de integración, teniendo en cuenta que conocen a fondo los procesos, áreas e indicadores de gestión del funcionamiento de la empresa, por lo cual puede determinar con mayor certeza el problema y resolverlo en menor tiempo. Por otro lado, además de los beneficios de la implementación de SIG en las pequeñas, medianas y grandes empresas, este proceso supone retos importantes para las organizaciones, como desplegar las actividades no solo a nivel nacional sino también a nivel internacional, teniendo en cuenta las crecientes exigencias del mercado, de manera, que se requieren diferentes estrategias que permitan evaluar cuál implementar de acuerdo con sus necesidades (Viloria, 2011).

Los aportes del presente artículo fundamentarán las necesidades de las empresas, de mejorar la satisfacción de los clientes, la estandarización de 
los procesos y procedimientos. Además, en el hecho de brindar a sus colaboradores una mayor seguridad laboral y una motivación por medio de la implementación de los sistemas de gestión ambiental y seguridad y salud en el trabajo (Icontec, 2017). De igual manera, en la necesidad de disminuir la documentación y lograr una verdadera integración de sus sistemas de gestión, para lo cual existen diferentes tipos de estrategias que hasta ahora han sido diseñadas de manera general y no han sido estandarizadas por tipo de empresa (Mosquera y Vásquez, 2015; Lleras, 2017; Pohludka y Štverková, 2019; Díaz y Rodríguez-Rojas, 2016; Stamou, 2003).

De acuerdo con los artículos incluidos en el análisis, se encontró que el $53 \%$ de los artículos han sido publicados en los últimos cinco años, el $34 \%$ en el período de 2010 a 2015 y un $13 \%$ entre 2002 y 2009. Además, se identificaron estudios en sectores particulares, tales como notariales, manufactura, siderúrgicas, ferreteros y energéticos. Con base en las variables definidas en la metodología en relación con SIG, modelos y SIG en pequeñas, medianas y grandes empresas, se describe cada una de ellas a continuación.

\section{Sistemas integrados de gestión}

Un sistema de gestión es un conjunto de procesos interconectados para lograr el resultado deseado con mayor eficacia. Junto con los aspectos básicos de las actividades, algunos otros son importantes para las organizaciones, por ejemplo, el medio ambiente, la seguridad y salud en el trabajo de los colaboradores, la responsabilidad social, etc. Así pues, un sistema de gestión está compuesto por todos los elementos que influyen en el rendimiento empresarial (Khanna et ál., 2009). Las investigaciones han demostrado que la gestión de la calidad, del medio ambiente y la seguridad 
y salud en el trabajo tienen una relación significativa con la calidad y el rendimiento financiero de las organizaciones (Khanna et ál., 2009). Algunas revisiones de la literatura en cuanto a los SIG indican que los principales temas investigados están relacionados con 1) los niveles de integración del SIG; 2) las principales motivaciones, beneficios y los obstáculos para operar múltiples sistemas de gestión; y 3) los modelos para la implementación de los SIG (Nunhes y Oliveira, 2020). Además, se destacaron las siguientes oportunidades de investigación futura: 1) investigar los niveles de integración desde una perspectiva a largo plazo; 2) considerar qué barreras a la integración de las auditorías pueden afectar posiblemente el grado de integración; 3) evaluar los resultados de la aplicación de los SIG mediante los resultados y objetivos empresariales; 4) realizar pruebas empíricas de los modelos propuestos para la aplicación de los SIG; y 5) analizar si los SIG pueden contribuir y, de qué manera, a que las empresas participen en la elaboración de las estrategias ambientalmente sostenibles (Nunhes y Oliveira, 2020).

Los temas de investigación futura en relación con los SIG permiten evidenciar, también, algunos de los problemas actuales de su implementación. Así, la integración a largo plazo y la integración de auditorías son, actualmente, algunos de los elementos que deben mejorarse para lograr la adecuada implementación de SIG en las empresas y mejorar su efectividad. Igualmente, la evaluación objetiva de los resultados y las pruebas empíricas de modelos para la aplicación de SIG constituyen un amplio campo de investigación en el que aún se quedan cortas las investigaciones respecto al tema de integración de sistemas de gestión en las empresas.

Dentro de los artículos revisados, se encontró que en los SIG implementados en la empresa las normas internacionales mayormente 
utilizadas para definir los sistemas calidad y ambiental son la ISO 9000 y la ISO 1400o, las cuales se considera que surgen teniendo en cuenta la necesidad de aportar mejoras tanto a la calidad de los productos, como de los procesos y de controlar los impactos ambientales, debido al enfoque de gestión de procesos que utilizan (Alzate-Ibañez et ál., 2018). La versión más actualizada de estas normas (2015) tiene sus principios en base a la calidad y el enfoque de procesos, tal como se mencionó anteriormente; además, se enfoca en la toma de decisiones que, a su vez, se basa en la experiencia y el liderazgo de las organizaciones. Estas dos normas en conjunto permiten incluir una cultura organizacional para la gestión estratégica de la calidad y el medio ambiente bajo su enfoque sistemático, así como aspirar al logro de la excelencia (Alzate-Ibañez et ál., 2018). Igualmente, existen perspectivas teóricas y empíricas para la integración de sistemas de gestión, las cuales han sido estudiadas en detalle (MoraContreras, 2019); a pesar de lo anterior, al hablar de normas para sistemas de gestión integrados de la familia ISO, no se tiene una norma que realice el compendio de los requisitos necesarios para su implementación (Bernardo et ál., 2009). Esta es una de las razones por las que las organizaciones aun encuentran dificultades para integrar dos o más sistemas de gestión; además, teniendo en cuenta que estos procesos demandan recursos humanos y financieros (Mora-Contreras, 2019; Nunhes y Oliveira, 2020). Lo cual indica que, en este campo, aunque se encuentran diversos estudios, aún queda mucho por conocer, detallar y desarrollar para ofrecer un marco de aportes que les permita a las empresas hacer uso de estas herramientas y lograr los impactos organizacionales esperados. 


\section{Modelos de sistemas integrados de gestión}

El proceso de integración no es fácil debido especialmente a las diferentes estructuras de los sistemas de gestión formalizados y su desarrollo como normas internacionales. Otro problema de este enfoque es el diseño, desarrollo y aplicación de un modelo genérico de gestión para la organización, a partir del cual, sobre la base de la integración de procesos y sistemas de gestión formalizados y no formalizados, se lleva a cabo la integración de la información, cuyo resultado es un modelo de gestión integrado (Majstorović y Marinković, 2011). Como se mencionaba anteriormente, el estudio de los SIG permite conocer diversas formas y estrategias de integración, en diferentes tipos de empresas y sectores; sin embargo, teniendo en cuenta la complejidad, tanto de la integración de sistemas de gestión como de las organizaciones mismas, en este campo aún falta detallar en mayor medida las estrategias más adecuadas y los elementos principales que se deben considerar.

En este sentido, la presente revisión permite determinar algunas de estas estrategias diferenciadas por tipo de empresa, por sector y describiendo las principales normas integradas. Dentro de los artículos analizados, se encontraron algunos modelos o estrategias de integración, en su mayoría sin tener un enfoque por tipo de empresa definido. Así, la tabla 2 muestra un resumen de los modelos o estrategias identificados. 
Tabla 5. Estrategias de integración de SIG identificadas

\begin{tabular}{|c|c|c|c|c|c|c|c|c|c|c|c|c|}
\hline \multirow{2}{*}{ N. ${ }^{\circ}$} & \multirow{2}{*}{ Autores } & \multirow{2}{*}{$\begin{array}{l}\text { Tamaño de la } \\
\text { organización }\end{array}$} & \multirow{2}{*}{ Sector } & \multicolumn{3}{|c|}{ SG Calidad } & \multirow{2}{*}{$\begin{array}{l}\text { SG Ambiental } \\
\text { ISO } 14001\end{array}$} & \multicolumn{4}{|c|}{ SG Seguridad } & \multirow{2}{*}{ Modelo } \\
\hline & & & & ISO 9001 & QS 9000 & TS 16949 & & OSHAS 18001 & ISO 18001 & DEC. 1072 & ISO 45001 & \\
\hline 1 & $\begin{array}{c}\text { Silva, C., } \\
\text { Magano, M, } \\
\text { Nogueira, T., } \\
\text { Dinis, M., Sousa, } \\
\text { H. }\end{array}$ & N/A & $\begin{array}{l}\text { Energético } \\
\text { combustibles } \\
\text { y cerámico }\end{array}$ & $x$ & & & $x$ & $x$ & & & $x$ & $\begin{array}{c}\text { Incluye elementos } \\
\text { necesarios para el } \\
\text { desarrollo de un } \\
\text { modelo de gestión } \\
\text { sostenible: las } \\
\text { estructura, como el } \\
\text { enfoque de procesos, } \\
\text { el ciclo PHVA, la } \\
\text { mejora continua, } \\
\text { ayudan a desarrollar } \\
\text { un negocio } \\
\text { sostenible de forma } \\
\text { eficaz y sistemática, } \\
\text { con mirada hacia el } \\
\text { futuro. }\end{array}$ \\
\hline 2 & $\begin{array}{c}\text { Algheriani, N.M., } \\
\text { Majstorovic, V.D, } \\
\text { Kirin, S., } \\
\text { Spasojevic } \\
\text { Brkic, V. }\end{array}$ & N/A & $\mathrm{N} / \mathrm{A}$ & $x$ & & & $x$ & $x$ & & & & $\begin{array}{c}\text { La estrategia de } \\
\text { integración abarca el } \\
\text { enfoque sistémico, } \\
\text { mejorando la } \\
\text { uniformidad y } \\
\text { homogeneidad del } \\
\text { SIG, el enfoque tecno } \\
\text { céntrico y la } \\
\text { promoción de } \\
\text { beneficios a nivel } \\
\text { operativo. }\end{array}$ \\
\hline 3 & $\begin{array}{c}\text { Laal, F.a, } \\
\text { Pouyakian, M.b, } \\
\text { Madvari, R.F.c, } \\
\text { Khoshakhlagh, } \\
\text { A.H.d,e, Halvani, } \\
\text { G.H }\end{array}$ & N/A & N/A & $x$ & & & $x$ & $x$ & & & & $\begin{array}{l}\text { Modelo de regresión } \\
\text { para determinar la } \\
\text { relación entre } \\
\text { sistemas. }\end{array}$ \\
\hline
\end{tabular}

Signos, Investigación en Sistemas de Gestión

ISSN: 2145-1389 | e-ISSN: 2463-1140 | DOI: https://doi.org/10.15332/24631140

Vol. 13 N. 02 | julio-diciembre de 2021 


\begin{tabular}{|c|c|c|c|c|c|c|c|c|c|c|c|c|}
\hline \multirow{2}{*}{$\mathrm{N}^{\circ}$} & \multirow{2}{*}{ Autores } & \multirow{2}{*}{$\begin{array}{l}\text { Tamaño de la } \\
\text { organización }\end{array}$} & \multirow{2}{*}{ Sector } & \multicolumn{3}{|c|}{ SG Calidad } & \multirow{2}{*}{$\begin{array}{l}\text { SG Ambiental } \\
\text { ISO } 14001\end{array}$} & \multicolumn{4}{|c|}{ SG Seguridad } & \multirow{2}{*}{ Modelo } \\
\hline & & & & ISO 9001 & QS 9000 & TS 16949 & & OSHAS 18001 & ISO 18001 & DEC. 1072 & ISO 45001 & \\
\hline 4 & $\begin{array}{l}\text { Campos, C.A.O., } \\
\text { Medeiros, D.D. }\end{array}$ & Pequeña . & Energético & $x$ & & & $x$ & & & & & $\begin{array}{l}\text { Modelo a partir de la } \\
\text { teoría general de } \\
\text { sistemas y un } \\
\text { modelo matemático } \\
\text { que permita la } \\
\text { integración de SG } \\
\text { parcial o totalmente. }\end{array}$ \\
\hline 5 & $\begin{array}{l}\text { Marieta Olarua } \\
\text { Dorin Maier } \\
\text { Diana Nicoarăc } \\
\text { Andreea Maier }\end{array}$ & N/A & $\mathrm{N} / \mathrm{A}$ & $x$ & & & $x$ & $x$ & & & & $\begin{array}{l}\text { Dos modelos de } \\
\text { estudio: evolutivo y } \\
\text { alineado, con } \\
\text { enfoque basado en } \\
\text { el ciclo PHVA. }\end{array}$ \\
\hline 6 & $\begin{array}{l}\text { Thaís Vieira } \\
\text { Nunhes y Otávio } \\
\text { José Oliveira }\end{array}$ & $\mathrm{N} / \mathrm{A}$ & $\mathrm{N} / \mathrm{A}$ & $x$ & & & $x$ & $x$ & & & & $\begin{array}{l}\text { Tendencia en } \\
\text { modelos, con base } \\
\text { en una revisión } \\
\text { bibliográfica de SIG } \\
\text { entre } 2005 \text { y } 2015 \text {. }\end{array}$ \\
\hline 7 & Rodríguez, A.L.L. & $\begin{array}{l}\text { Pequeñas y } \\
\text { medianas. }\end{array}$ & $\mathrm{N} / \mathrm{A}$ & $x$ & & & $x$ & $x$ & & & & $\begin{array}{c}\text { Modelo de } \\
\text { integración con base } \\
\text { en el Balance Score } \\
\text { Card (BSC). }\end{array}$ \\
\hline 8 & $\begin{array}{l}\text { Jaudová, J., } \\
\text { Zelený, J., } \\
\text { Hroncová } \\
\text { Vicianová, J., } \\
\text { Marková, I. }\end{array}$ & $\begin{array}{l}\text { Pequeñas y } \\
\text { medianas. }\end{array}$ & Maderero & $x$ & & & $x$ & $x$ & & & & $\begin{array}{l}\text { No describe los } \\
\text { modelos y analiza los } \\
\text { existentes en las } \\
\text { empresas. }\end{array}$ \\
\hline 9 & Rönninger, S. & Pequeña & Químico & $x$ & & & $x$ & & & & & $\begin{array}{l}\text { Describe el modelo } \\
\text { de SGI de la empresa } \\
\text { de análisis en el } \\
\text { sector químico. }\end{array}$ \\
\hline
\end{tabular}

Signos, Investigación en Sistemas de Gestión

ISSN: 2145-1389 | e-ISSN: 2463-1140 | DOI: https://doi.org/10.15332/24631140

Vol. 13 N. ${ }^{\circ} 2$ | julio-diciembre de 2021 


\begin{tabular}{|c|c|c|c|c|c|c|c|c|c|c|c|c|}
\hline \multirow{2}{*}{ N. ${ }^{\circ}$} & \multirow{2}{*}{ Autores } & \multirow{2}{*}{$\begin{array}{l}\text { Tamaño de la } \\
\text { organización }\end{array}$} & \multirow{2}{*}{ Sector } & \multicolumn{3}{|c|}{ SG Calidad } & \multirow{2}{*}{$\begin{array}{l}\text { SG Ambiental } \\
\text { ISO } 14001\end{array}$} & \multicolumn{4}{|c|}{ SG Seguridad } & \multirow{2}{*}{ Modelo } \\
\hline & & & & ISO 9001 & QS 9000 & TS 16949 & & OSHAS 18001 & ISO 18001 & DEC. 1072 & ISO 45001 & \\
\hline 10 & $\begin{array}{l}\text { Pun, M., } \\
\text { Wilcock, A., } \\
\text { Aung, M. }\end{array}$ & $\begin{array}{l}\text { Pequeñas, } \\
\text { medianas y } \\
\text { grandes. }\end{array}$ & Alimentos & $x$ & & & & & & & & $\begin{array}{c}\text { El mayormente } \\
\text { utilizado es el } \\
\text { HACCP. }\end{array}$ \\
\hline 11 & $\begin{array}{l}\text { Gangolells, M., } \\
\text { Casals, M. }\end{array}$ & N/A & Construcción & & & & $x$ & $x$ & & & & $\begin{array}{c}\text { Integra los sistemas } \\
\text { de salud y seguridad } \\
\text { con el sistema } \\
\text { ambiental en } \\
\text { empresas } \\
\text { constructoras, con } \\
\text { enfoque ontológico } \\
\text { de definición de } \\
\text { impactos } \\
\text { ambientales y } \\
\text { riesgos en salud. }\end{array}$ \\
\hline 12 & $\begin{array}{l}\text { Fabrício, C. } \\
\text { Poltronieri, G. } \\
\text { Miller, D. Ganga, } \\
\text { M. Gerolamo, C. }\end{array}$ & N/A & $\mathrm{N} / \mathrm{A}$ & $x$ & & & $x$ & $x$ & & & & $\begin{array}{c}\text { Relación entre la } \\
\text { integración de los } \\
\text { sistemas y el } \\
\text { desarrollo sostenible, } \\
\text { basado en la } \\
\text { madurez y } \\
\text { sostenibilidad de las } \\
\text { organizaciones. }\end{array}$ \\
\hline 13 & $\begin{array}{l}\text { Bernal-Conesa, } \\
\text { J. Briones- } \\
\text { Peñalver, A. } \\
\text { Nieves-Nieto, C. }\end{array}$ & N/A & Tecnológico & $x$ & & & $x$ & $x$ & & & & $\begin{array}{c}\text { Análisis estadístico } \\
\text { para determinar la } \\
\text { relación entre la } \\
\text { integración de } \\
\text { sistemas de gestión y } \\
\text { la RSE. }\end{array}$ \\
\hline 14 & $\begin{array}{l}\text { José Stalin } \\
\text { Rojas Amaya }\end{array}$ & N/A & Transporte & $x$ & & & $x$ & $x$ & & & & $\begin{array}{c}\text { Describe la } \\
\text { integración de los } \\
\text { SIG ISO y el sistema } \\
\text { de gestión BASC por } \\
\text { medio del ciclo PHVA. }\end{array}$ \\
\hline
\end{tabular}

Signos, Investigación en Sistemas de Gestión

ISSN: 2145-1389 | e-ISSN: 2463-1140 | DOI: https://doi.org/10.15332/24631140

Vol. 13 N. 02 | julio-diciembre de 2021 


\begin{tabular}{|c|c|c|c|c|c|c|c|c|c|c|c|c|}
\hline \multirow{2}{*}{ N. ${ }^{\circ}$} & \multirow{2}{*}{ Autores } & \multirow{2}{*}{$\begin{array}{l}\text { Tamaño de la } \\
\text { organización }\end{array}$} & \multirow{2}{*}{ Sector } & \multicolumn{3}{|c|}{ SG Calidad } & \multirow{2}{*}{$\begin{array}{l}\text { SG Ambiental } \\
\text { ISO } 14001\end{array}$} & \multicolumn{4}{|c|}{ SG Seguridad } & \multirow{2}{*}{ Modelo } \\
\hline & & & & ISO 9001 & QS 9000 & TS 16949 & & OSHAS 18001 & ISO 18001 & DEC. 1072 & ISO 45001 & \\
\hline 15 & $\begin{array}{l}\text { Weyandta, A. } \\
\text { Reis da Costab, } \\
\text { S. Nunesc, M. } \\
\text { Gaspara, A. }\end{array}$ & N/A & Pesquero & & & & $x$ & & & & & $\begin{array}{c}\text { Describe las } \\
\text { motivaciones de la } \\
\text { integración, no se } \\
\text { desarrolla un modelo } \\
\text { de integración } \\
\text { especifico. }\end{array}$ \\
\hline 16 & $\begin{array}{c}\text { Rodríguez } \\
\text { Rodríguez, E.; } \\
\text { Montenegro } \\
\text { Narváez, C. }\end{array}$ & Pequeña & Ferretero & $x$ & & & & & & $x$ & & $\begin{array}{l}\text { Comparación Pass } \\
99 \text { y UNE } 66177 \\
\text { Selección de la } \\
\text { estructura de alto } \\
\text { nivel en combinación } \\
\text { con el ciclo PHVA. }\end{array}$ \\
\hline 17 & $\begin{array}{l}\text { Alzate- Ibáñez, } \\
\text { A; Ramírez Ríos, } \\
\text { J; Bedoya } \\
\text { Montoya, L. }\end{array}$ & N/A & Siderúrgico & $x$ & & & $x$ & & & & & $\begin{array}{l}\text { Enfoque sistémico de } \\
\text { procesos, basado en } \\
\text { la estructura de alto } \\
\text { nivel y el ciclo PHVA. }\end{array}$ \\
\hline 18 & $\begin{array}{l}\text { Malagón } \\
\text { Medina, } \\
\text { Angélica }\end{array}$ & $\mathrm{N} / \mathrm{A}$ & N/A & $x$ & & & $x$ & $x$ & & & & $\begin{array}{c}\text { Revisión de varios } \\
\text { artículos y } \\
\text { determinación de } \\
\text { estrategias } \\
\text { nombrada y sin } \\
\text { nombrar, tales como: } \\
\text { • Enfoque y } \\
\text { principios TQM- } \\
\text { EFQM. } \\
\text { • Enfoque de } \\
\text { sistemas. } \\
\text { - Enfoque por } \\
\text { procesos. } \\
\text { - Indicadores de } \\
\text { integración. }\end{array}$ \\
\hline
\end{tabular}

Signos, Investigación en Sistemas de Gestión

ISSN: 2145-1389 | e-ISSN: 2463-1140 | DOI: https://doi.org/10.15332/24631140

Vol. 13 N. 02 | julio-diciembre de 2021 


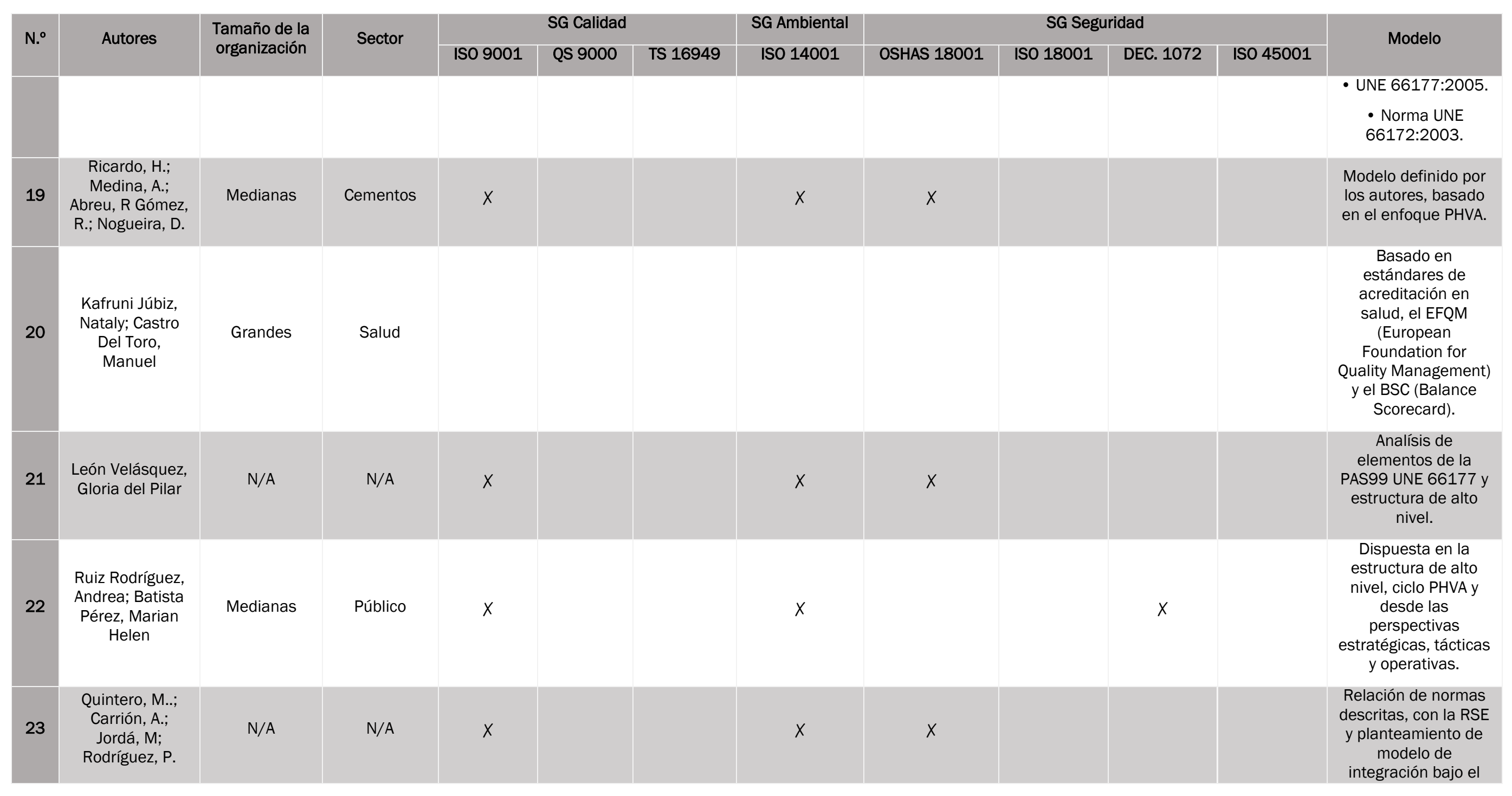

Signos, Investigación en Sistemas de Gestión

ISSN: 2145-1389 | e-ISSN: 2463-1140 | DOI: https://doi.org/10.15332/24631140

Vol. 13 N. ${ }^{\circ} 2$ | julio-diciembre de 2021 


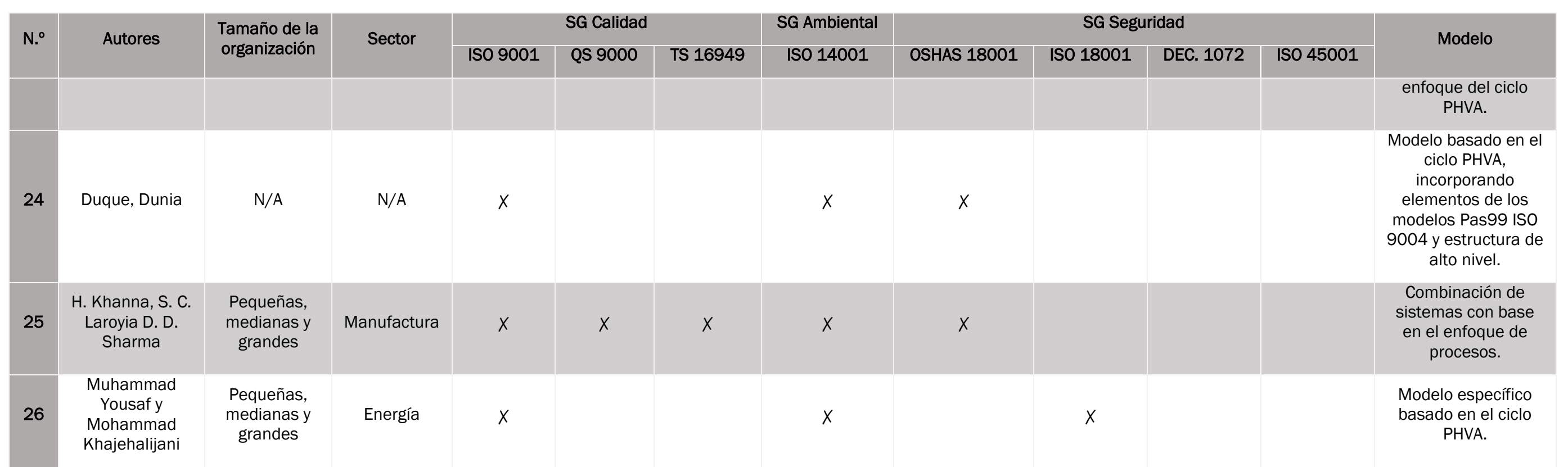

Fuente: elaboración propia a partir de información de bases de datos.

Signos, Investigación en Sistemas de Gestión

ISSN: 2145-1389 | e-ISSN: 2463-1140 | DOI: https://doi.org/10.15332/24631140

Vol. 13 N. 02 | julio-diciembre de 2021 
De acuerdo con los resultados encontrados, de los 55 artículos analizados, 26 (47\%) describen modelos o estrategias en relación con la integración de sistemas de gestión. Estos modelos o estrategias se distribuyen en cuanto al tamaño de la empresa y tienen en cuenta que, de los 26 artículos presentados en la tabla anterior, 11 mencionan diferencias entre empresas de distintos tamaños. En este sentido, la tabla 3 muestra el tipo de estrategias y de normas usadas para la integración de sistemas en cada tamaño de empresa.

Tabla 6. Estrategias de integración de SIG identificadas por tamaño de organización

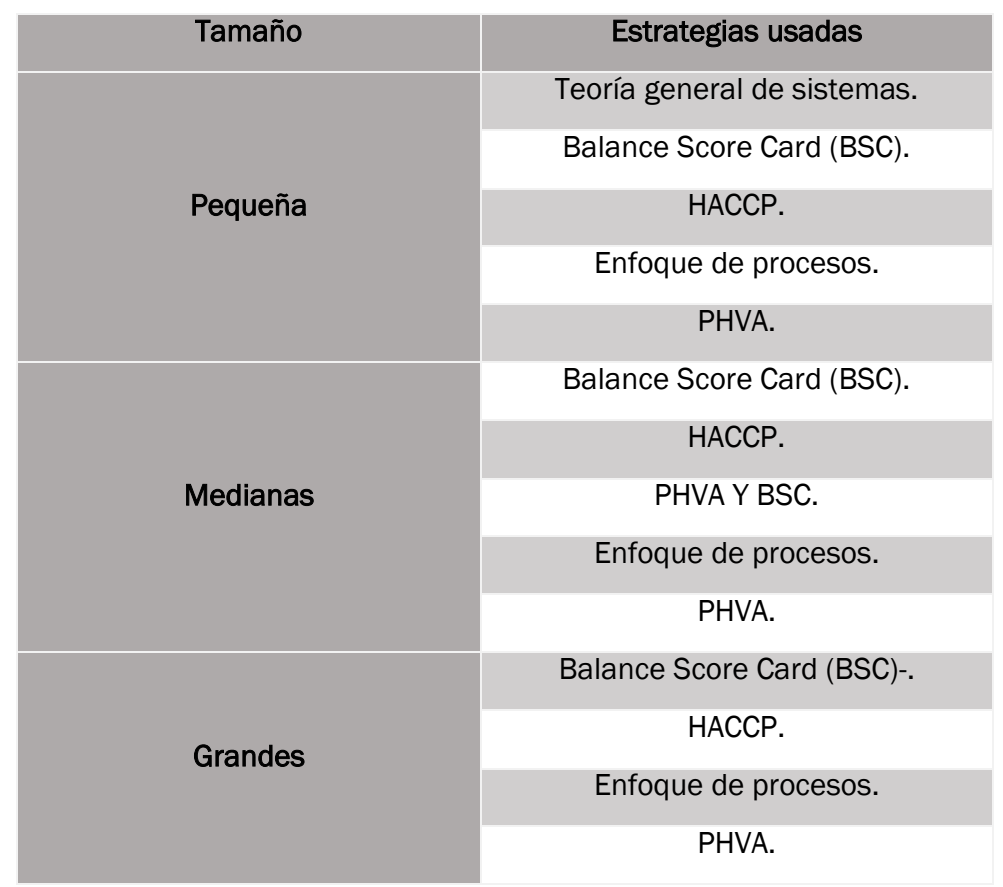

Fuente: elaboración propia (2021), fundamentada en información de bases de datos.

Se observa que las estrategias más usadas son PHVA, HACCP, Balance Score Card y el enfoque de procesos, en los tres tamaños de empresas analizados; sin embargo, en las pequeñas empresas también se han utilizado el enfoque basado en teoría de sistemas. En cuanto a los sectores 
en los que se han diseñado y utilizado estos modelos o estrategias, se encuentran principalmente energía con un 19 \% y madera, químicos, alimentos, construcción, tecnologías, transporte, pesquero, ferretero, siderúrgica, cementos, salud, público y manufactura con un $6 \%$ respectivamente.

\section{Conclusiones}

La principal conclusión del presente artículo se enfoca en el análisis de las estrategias de integración encontradas en la literatura revisada, en donde se encontró que solamente 11 de los 26 artículos enfocan las estrategias de acuerdo con el tamaño de las organizaciones, las demás estrategias de integración planteadas se centran en determinar los requisitos y los enfoques más adecuados, sin tener en cuenta el tamaño. Dentro de las principales estrategias de integración encontradas se observan aquellas basadas en el ciclo PHVA, Balance Score Card (BSC) y enfoque de procesos, también se encuentran estrategias basadas en estructuras de alto nivel y enfoque de sistemas.

El resultados del análisis bibliométrico, acerca de las estrategias de integración, permitió identificar que, para las pequeñas empresas las estrategias más usadas son la teoría general de sistemas, el BSC, el HACCP, el enfoque de procesos (que se utiliza en las normas ISO) y el ciclo PHVA; esto indica que las pequeñas y medianas empresas tienen particularmente un enfoque general de la organización y que utilizan estrategias reconocidas y no diseños de modelos especializados en cada tipo de empresa.

Con respecto al objetivo del presente artículo, es posible concluir que las estrategias de integración de los sistemas de gestión en las empresas no presentan diferencias significativas en cuanto al tamaño de la empresa, sino que permiten evidenciar que se enfocan en diversos sectores de la 
economía, tales como energía, combustibles, cerámica, alimentos, medicamentos, metalmecánico, madera, químico, construcción, tecnologías, transporte, pesquero, bibliotecas, notarial, ferretero, siderúrgica, manufactura, cementos, salud, público. Esto permite concluir que los modelos de SIG pueden estar mayormente diseñados en función del sector y no del tamaño de la empresa.

Finalmente, es importante concluir que el acceso abierto a la información presentó restricciones en los artículos que fueron encontrados en las bases de datos, relacionados con el tema de búsqueda, por lo cual el análisis de resultados evidenció limitaciones en la posibilidad de describir otras estrategias utilizadas por las pequeñas, medianas y grandes empresas para la integración de los sistemas de gestión.

\section{Referencias}

Alzate-Ibañez, A. M., Ramírez Ríos, J. F., y Bedoya Montoya, L. M. (2018). Modelo para la implementación de un sistema integrado de gestión de calidad y ambiental en una empresa Siderúrgica. Ciencias Administrativas, 13, 032.

https://doi.org/10.24215/23143738e032

Algheriani, N. M., Majstorovic, V.D, Kirin, S., y Spasojevic Brkic, V. (2019). Risk Model for Integrated Management System. Tehnički vjesnik, 26(6), 1833-1840.

Arévalo, N., y Molano, J. (2013). De la salud ocupacional a la gestión de la seguridad y salud en el trabajo: más que semántica, una transformación del sistema general de riesgos laborales. Innovar, 23(48), 21-32.

https://www.redalyc.org/articulo.oa?id=81828690003

Bernal-Conesa, J., Briones-Peñalver, A. y Nieves-Nieto, C. (2016). The integration of CSR management systems and their influence on the performance of technology companies. European Journal of Management and Business Economics, 25, 121132. 
Brenes, J. A. A., Arias, A. M., y Sánchez, E. V. (2017). Development of an integrated management system for micro, small and medium enterprises based on the standard INTE 01-01-09: 2013. Tec Empresarial, 11(2), 17-26.

https://www.scielo.sa.cr/scielo.php?pid=S1659$33592017000200017 \&$ script $=$ sci abstract\&tlng=en

Campos, C. A. O., y Medeiros, D. D. (2009). A model for integration of management systems. Produção, 70-86.

Díaz, C. C. R., y Rodríguez-Rojas, Y. L. (2016). Beneficios e impactos de la implementación de normas técnicas en las organizaciones: Una revisión sistemática. Signos, Investigación en Sistemas de Gestión, 8(2), 133-167. https://doi.org/10.15332/s2145-1389.2016.0002.07

Deming, E., y Medina, J. N. (1989). Calidad, productividad y competitividad: la salida de la crisis. Díaz de Santos.

Duque, D. (2017). Modelo teórico para un sistema integrado de gestión (seguridad, calidad y ambiente). Ingeniería Industrial. Actualidad y Nuevas Tendencias, 18 , 115-130. https://www.redalyc.org/pdf/2150/215052403009.pdf

Etkin, J. (2003). Gestión de la complejidad en las organizaciones. Oxford University Press.

Gangolells, M., y Casals, M. (2012). An ontology-based approach for on-site integrated environmental and health and safety management. Revista Ingeniería de Construcción, 27(3), 103-127. https://upcommons.upc.edu/handle/2117/17362

García de la Torre, C. (2001). Una aproximación a los estudios interculturales en la admininstración. Administración y Organizaciones, 6(3), 35-58.

García, F., Ibáñez, J., y Francisco, A. (2000). El análisis de la realidad social: métodos y técnicas de investigación. Alianza.

Gómez, M. A. B. (2009). Propuesta de un sistema integrado de gestión para una pyme caso compañía high safety networks -HSN-SAS [tesis de pregrado]. Corporación Universitaria Minuto de Dios. http://hdl.handle.net/10656/1656

González González, A., y Fernández, E. M. (2000). La cultura de la organización en la gestión total de la calidad. Ensaios e Ciencia:Ciencias Biológicas, Agrarias e da Saúde, 4(3), 99-114. http://www.redalyc.org/articulo.oa?id=26040307 
Goodenough, W. H. (1957). Componential Analysis, Kinship studies in cultural anthropology are producing a new tool for semantic analysis. New Series, 156(3779), 132-167. https://www.jstor.org/stable/1721317

Gorbea-Portal, S. (2013). Tendencias transdisciplinarias en los estudios métricos de la información y su relación con la gestión de la información y del conocimiento. Perspectivas em Gestão \& Conhecimento, 3(1), 13-27. https://dialnet.unirioja.es/servlet/articulo? codigo $=4800992$

Hernández, R., Fernández, C., y Baptista, M. del P. (2010). Metodología de la investigación (5 ed.). Mc Graw Hill.

https://www.esup.edu.pe/descargas/dep investigacion/Metodologia\%20de\%20la \%20investigaci\%C3\%B3n\%205ta\%20Edici\%C3\%B3n.pdf

Ibañez, J. (2000). Perspectivas de la investigación social: el diseño en las tres perspectivas. En F. M. García, J. Ibáñez, y F. Alvira (Coords.), El análisis de la realidad social: métodos y técnicas de investigación ( $3 \cdot^{\mathrm{a}}$ ed.). Alianza.

Icontec. (2017). Norma Técnica Colombiana NTC 6001:2017. Modelo de gestión para micro empresa y pequeñas empresa (Mypes). Icontec.

Israr, M., y Gangele, A. (2014, 7-9 de enero). A Comparative Analysis between Small and Medium Scale Manufacturing Company through Total Quality Management Techniques. Proceedings of the 2014 International Conference on Industrial Engineering and Operations Management Bali, Indonesia.

Jaudová, J., Zelený, J., Hroncová Vicianová, J., y Marková, I. (2015). Stakeholder Management as Part of Integrated Management System in the Furniture Industry. Procedia Economics and Finance, 34, 129-133. https://doi.org/10.1016/S22125671(15)01610-X

Jiménez, E. (2014). Bases para un concepto de las “-metrías”. Cognósfera.

Kafruni J. N., y Castro Del Toro, M. (2018). Sistema integrado de gestión para las IPS de alta complejidad de la ciudad de Barranquilla basado en los estándares de acreditación en salud, el modelo European foundation for quality management y el Balanced Scorecard. Prospectiva, 16(1), 91-99.

http://www.scielo.org.co/scielo.php?script=sci abstract\&pid=S1692$\underline{82612018000100091 \& \operatorname{lng}=\text { es\&nrm=iso }}$ 
Khanna, H., Laroyia, S. C., y Sharma, D. D. (2009). A survey on Indian Experience on Integrated Management Standards (IMS). International Journal for Quality Research, 3(3). https://journals.indexcopernicus.com/api/file/viewByFileId/230035.pdf

Kuznik, A., Hurtado Albir, A., y Espinel Berenguer, A. (2010). El uso de la encuesta de tipo social en Traductología. MonTi. Monografías de Traducción e Interpretación, 2. http://www.redalyc.org/articulo.oa?id=265119729015

Laal, F., Pouyakian, M., Madvari, R. F., Khoshakhlagh , A. H., y Halvani, G. H. (2019). Investigating the Impact of Establishing Integrated Management Systems on Accidents and Safety Performance Indices: A Case Study. Safety and Health at Work, 10(1), 54-6o. https://doi.org/10.1016/j.shaw.2018.04.001

León, G. (2018). Análisis de percepción de la integración de sistemas de gestión. Signos, Investigación en Sistemás de Gestión, 10(1), 139-156. https://doi.org/10.15332/s2145-1389.2018.0001.08

Lleras, S. D. (2017). Propuesta de un modelo de gestión integral para aporte a la estrategia de Sifer S.A, basado en la ISO 9001:2015 y la NTC 5801:2008. 108 [tesis de maestría]. Universidad Externado de Colombia. https://bdigital.uexternado.edu.co/handle/o01/300

López, A. L. (2009). Sistema integrado de gestión para empresas turísticas. Estudios Gerenciales, 25(112), 131-150. https://doi.org/10.1016/S0123-5923(09)70083-X

Malagón, A. (2018). Revisión sistemática de teorías de integración de sistemas de gestión normalizados. Signos, Investigación en Sistemás de Gestión, 1O(1), 177-191. https://doi.org/10.15332/s2145-1389.2018.0001.10

Majstorović, V., y Marinković, V. (2011). The development of business standardization and integrated management systems. Journal of Biomedical Chemistry, 3o(4), 334-345. https://doi.org/10.2478/v10011-011-0015-5

Michán, L., y Muñoz-Velasco, I. (2013). Cienciometría para ciencias médicas: Definiciones, aplicaciones y perspectivas. Investigación en Educación Médica, 2(6), 100-106. https://www.redalyc.org/pdf/3497/349733227006.pdf

Misas Arango, G. (2004). La educación superior en Colombia. Análisis y estrategias para su desarrollo. Universidad Nacional de Colombia.

Signos, Investigación en Sistemas de Gestión

ISSN: 2145-1389 | e-ISSN: 2463-1140 | DOI: https://doi.org/10.15332/24631140

Vol. 13 N.० 2 | julio-diciembre de 2021 
Mora-Contreras, R. (2019). Sistemas integrados de gestión de las normas ISO 9001 e ISO 30301 en el contexto notarial colombiano. Estudios Gerenciales, 35(115) https://doi.org/10.18046/j.estger.2019.151.3248

Muhammad, Y., y Mohammad K. (2011). Design and implementation of integrated management system [tesis de maestría]. Mälardalen University Sweden.

Munduate, L. (1997). Psicología social de la organización. Las personas organizando. Pirámide.

Nunhes, T. V., y Oliveira, O. J. (2020). Analysis of Integrated Management Systems research: Identifying core themes and trends for future studies. Total Quality Management \& Business Excellence, 31(11-12), 1243-1265. https://doi.org/10.1080/14783363.2018.1471981

OIT. (2019, 10 de octubre). La mayor parte del empleo mundial procede de las pequeñas empresas y los trabajadores independientes, concluye un nuevo informe de la OIT. OIT. http://www.ilo.org/global/about-theilo/newsroom/news/WCMS 723414/lang--es/index.htm

Olaru, M., Maier, D., Nicoară, D., y Maier, A. (2014). Establishing the basis for Development of an Organization by Adopting the Integrated Management Systems: Comparative Study of Various Models and Concepts of Integration. Procedia Social and Behavioral Sciences, 109, 693-697. https://doi.org/10.1016/j.sbspro.2013.12.531

Oliveros, C., y Soto, M. (2016). Planeación orientada a la implementación de un sistema integrado de gestión en una pyme. Dinamica ambiental, 1, 45-58. https://doi.org/10.18041/2590-6704/ambiental.1.2016.4590

Omar, A., y Florencia Urteaga, A. (2010). El impacto de la cultura nacional sobre la cultura organizacional. Universitas Psychologica, 9(1), 79-92. http://www.scielo.org.co/scielo.php?pid=S165792672010000100007\&script $=$ sci abstract\&tlng=es

Ospina, S. N., y Rivera, V. (2013). Documentación del modelo de gestión para micro y pequeñas empresas ntc-6oo1 en la empresa bienes Y Finanzas Servicios Integrales S.A.S en Armenia, Quindío [tesis de especialización]. Universidad Tecnológica de Pereira. http://hdl.handle.net/11059/4960 
Parada, S. M. S., y Paredes, S. J. M. (2014). Propuesta de preparación para la implementación de un sistema de gestión integrado orientada a las pymes de Medellín y el área metropolitana [tesis doctoral]. Universidad Pontificia Bolivariana. http://hdl.handle.net/20.500.11912/2246

Paramo Morales, D. (2001). Hacia la construcción de un modelo de cultura organizacional orientada al mercado. Revista Colombiana de Marketing, 2(2), 126. http://www.redalyc.org/articulo.oa?id=10900206

Pohludka, M., y Štverková, H. (2019). The Best Practice of CRM Implementation for Small- and Medium-Sized Enterprises. Administrative Sciences, 9(1), 22. https://doi.org/10.3390/admscig010022

Poltronieri, F., Miller, G., Ganga, D., y Gerolamo, C. (2019). Maturity in management system integration and its relationship with sustainable performance. Journal of Cleaner Production, 207(10), 236-247. https://doi.org/10.1016/j.jclepro.2018.09.250

Pun, M., Wilcock, A., y Aung, M. (2007). Experience and perceptions of ISO 9000 and HACCP by Hong Kong food and beverage organizations. Journal of Asia Business Studies, 1(2), 67-76. https://doi.org/10.1108/15587890780001297

Quintero, M., Carrión, A., Jordá, M., y Rodríguez, P. (2015). La responsabilidad social empresarial y el modelo de excelencia EFQM como marco de integración de los sistemas de gestión en las organizaciones. Ingeniería y Competitividad, 17(1), 9-22. https://doi.org/10.25100/iyc.v17i1.2197

Reyes, R., Ibáñez, J., y Álvarez Uría, F. (1992). Las ciencias sociales en España. Historia inmediata, críticas y perspectivas. Complutense.

Riaño, M. (2009). Gestión de la seguridad y salud en el trabajo en hos-pitales públicos bogotanos de alta complejidad: Una perspectiva estratégica. Universidad Nacional de Colombia.

Ricardo, H., Medina, A., Abreu, R., Gómez, R., y Nogueira, D. (2018). Modelo para la mejora de procesos en contribución a la integración de sistemas. Ingeniería Industrial, 34(1), 15-23. http://scielo.sld.cu/pdf/rii/v39n1/riio3118.pdf

Rodríguez Garay, R. (2009). La cultura organizacional, un potencial estratégico desde la perspectiva de la administración. Invenio, 12(22), 67-92. https://www.redalyc.org/pdf/877/87722106.pdf 
Rodríguez, E. R. R., y Montenegro, C. (2019). Metodología para la integración de la gestión en una pyme del sector ferretero en Colombia. Signos, Investigación en Sistemas de Gestión, 11(2), 83-98. https://doi.org/10.15332/24631140.5083

Rodríguez Gutiérrez, J. K., Gómez Velasco, N. Y., y Herrera-Martínez, Y. (2017). Técnicas bibliométricas en dinámicas de producción científica en grupos de investigación. Caso de estudio: Biología-UPTC. Revista Lasallista de Investigación, 14(2), 73-82. https://doi.org/10.22507/rli.v14n2a7

Rojas, J. (2014). Elementos para la integración de sistemas de gestión y su importancia en la cadena productiva del transporte de carga terrestre en Colombia. Suma de negocios, 5(12), 136-142. https://doi.org/10.1016/S2215-910X(14)70035-7

Rönninger, S. (2002). Implementation of a Process-Oriented ISO 9000 and ISO 14000 Management System in a Chemical Manufacturing Site. Chimia International Journal for Chemistry, 56(12), 714-720. https://doi.org/10.2533/000942902777679894

Rosales Ortíz, R. (1997). Estilos de dirección y clima organizacional. Revista de ciencias sociales, $77,141-154$.

Ruiz, A., y Batista, M. (2018). Integración de los sistemas de gestión de calidad, ambiental y seguridad y salud en el trabajo en una entidad pública del orden nacional del sector hacienda. Signos, Investigación en Sistemás de Gestión, 1O(1), 141-157. https://doi.org/10.15332/s2145-1389.2018.0002.08

Sánchez Manchola, I. D. (2008). Los estilos de dirección y liderazgo, propuesta de un modelo de caracterización y análisis. Pensamiento y Gestión, 25, 1-39. https://rcientificas.uninorte.edu.co/index.php/pensamiento/article/view/3194/49 31

Silva, C., Magano, M, Nogueira, T., Dinis, M., y Sousa, H. (2020). Sustainable Management Systems Standards (SMSS): Structures, Roles, and Practices in Corporate Sustainability. Sustainability, 12.

http://dx.doi.org/10.3390/su12155892

Soria Romo, R. (2008). Comunicación organizacional: un modelo aplicable a la microempresa. Revista Científica Teorías, Enfoques y Aplicaciones en las Ciencias Sociales, 1(1), 9-17. https://dialnet.unirioja.es/servlet/articulo?codigo=3990506 
Stamou, T. (2003). Integrated Management Systems in Small Medium-Sized Enterprises: Theory and Practice [tesis de maestría]. University of East Angila. https://citeseerx.ist.psu.edu/viewdoc/download?doi=10.1.1.534.8750\&rep=rep1\&t ype $=$ pdf

Toro Alvarez, F. (2001). El clima organizacional. Perfil de empresas colombianas. Cincel.

Viloria, S. G. (2011). Sistemas integrados de gestión, un reto para las pequeñas y medianas empresas. Escenarios, 9(1), 69-89. https://dialnet.unirioja.es/servlet/articulo? codigo $=387524$

Weyandta, A., Reis da Costab, S., Nunesc, M. y Gaspara, A. (2011). Environmental \& food safety management systems, according to ISO 14001 \& ISO 22000 in fish processing plants: experiences, critical factors \& possible future strategies. Procedia Food Science, 1, 1901-1906. https://doi.org/10.1016/j.profoo.2011.09.279 